\title{
Research on the Treatment of Colorectal Cancer Based on Network Pharmacology and Molecular Docking
}

\section{Xinyao Shu}

Chengdu University of Traditional Chinese Medicine

Yunwu Zhang ( $\nabla$ zhangyunwu@stu.scu.edu.cn )

Sichuan University

\section{Yujia Wang}

Chengdu University of Traditional Chinese Medicine

\section{Jing Wu}

Chengdu University of Traditional Chinese Medicine

\section{Xingyu Chen}

Chengdu University of Traditional Chinese Medicine

\section{Ruitong Xu}

Chengdu University of Traditional Chinese Medicine

Xingting Liu

Chengdu University of Traditional Chinese Medicine

Jingsong Luo

Chengdu University of Traditional Chinese Medicine

\section{Tingting Deng}

Chengdu University of Traditional Chinese Medicine

\section{Research Article}

Keywords: Sophora flavescens, decoction, colorectal cancer, network pharmacology, molecular docking

Posted Date: March 25th, 2021

DOI: https://doi.org/10.21203/rs.3.rs-263797/v2

License: (c) (1) This work is licensed under a Creative Commons Attribution 4.0 International License. Read Full License 


\section{Abstract}

Background: To predict the mechanism of Huaijiao Diyu Decoction in the treatment of colorectal cancer.

Methods: The active components and related targets of 7 kinds of Chinese medicinal materials were collected from the database of Chinese medicinal materials. CRC related targets are obtained from the Genes card database. cross targets of disease and drug targets were input into the STRING database to construct protein-protein interaction networks.GO enrichment analysis and KEGG enrichment analysis using clusterProfiler packets in the language, Finally, the interaction between core components and core targets is discussed by molecular docking.

Result: TCMSP database prompts, There are 34 active compounds mapping 114 targets in Sophora japonica decoction; $\mathrm{G}$ enecards database prompts, A total of 858 targets are closely related to $\mathrm{CRC}$, The two data sets map each other to obtain 114 intersection targets. Core components of Sophora flavescens decoction in treating CRC may be quercetin, kaempferol, luteolin, The core therapeutic targets may be PTGS2ロPTGS1DHSP90AA1 and AR, CRC related pathways involve multiple molecular functions such as cell proliferation, apoptosis, cell signal transduction, metabolism, endocrine, tumor immunity, transcription and cell metabolism. Molecular docking results show that the binding ability of PTGS10 PTGS2 $\triangle A R$ to core components is strong, The Vina value (binding energy) of the interaction between PTGS1 protein and core components is the best, $-7.9 \mathrm{kcal} / \mathrm{mol}$.

Conclusion: This study demonstrates the mechanism of multi-component, multi-target and multi-pathway action in the treatment of CRC, Can provide the idea for clarifying the specific mechanism of Huaijiao Diyu decoction in the treatment of $\mathrm{CRC}$ in the future.

\section{Background}

Colorectal cancer is one of the most common malignancies in gastrointestinal tract[1] , typical symptoms are hematochezia, abdominal pain, abdominal mass, weight loss, anemia, intestinal obstruction [2]. According to the latest report in 2018, globally, the incidence of colorectal cancer ranks fourth among malignant tumors, death rate ranked second [3]. January 2019, The National Cancer Center released the latest issue of national cancer statistics, the incidence and mortality of colorectal cancer in China ranked third and fifth respectively, The incidence of colorectal cancer in China is on the rise. The western medicine treatment of colorectal cancer is still mainly surgical treatment. And the only cure for colorectal cancer, Clinical use of surgery, radiotherapy, chemotherapy, targeted therapy and other treatment methods combined, fluorouracil (5 FU) based regiments and oxaliplatin were generally accepted as a standard adjuvant regimen [4]. But since most patients are diagnosed as advanced, Difficult treatment, Poor prognosis or long-term radiotherapy and chemotherapy lead to a serious decline in the quality of life of patients, Produce serious adverse reactions.

Traditional Chinese medicine is an important tumor-assisted treatment [5]. A number of studies have shown that Chinese medicine has played a positive role in blocking tumor energy metabolism, inducing 
cancer cell apoptosis, reducing tumor cell recurrence and metastasis rate, and inhibiting tumor cell growth and value-added, surgery,chemotherapy and taking Chinese herbal decoction were protective factors for $\mathrm{CRC}$ recurrence and metastasis has a positive effect on the survival time of CRC patients [6,7]. TCM believes that colorectal cancer has spleen and kidney yang deficiency, qi and blood deficiency, spleen deficiency dampness Sheng, spleen deficiency deficiency and other syndromes. Clinical practice has proved that Huaijiao Diyu decoction has a significant effect in the treatment of colorectal cancer, but its treatment mechanism is not clear and needs further exploration.

Sophora flavescens decoction from the "political criteria ", for the main prescription for clearing heat and cooling blood, this prescription by Sophora flavescens, Radix Paeoniae Alba, Gardenia, Fructus Aurantii, Scutellariae, Schizonepeta. A result suggest that pretreatment with noncytotoxic concentrations of fructus sophorae extract (FSE) xhibitsanti-

inflamatoryactivitybyinhibitinginflammatorymediaandcytokinesthroughtheinactivationofNF-KB, ERK and JNK, and it may offer therapeutic potential for creating inflammatory diseases associated with macroactivity [8]. The present results suggest that FSE may protect against inflammation and bone damage, and would be a valuable candidate for further investigation as a novel anti-arthritic agent [9]. Data suggested that the addition of SOWPa (from Sanguisorba officialis L. Rosaceae) to PRP increased the reproductive capacity of ACL fibers by blocking the TLR-4/NF-K B pathway. The addition of sowpa to prp can improve the regeneration ability of acl fibroblasts by blocking the $t \mathrm{r}-4 / \mathrm{nfb}$ pathway.components from Sanguisorba officinalis L.on Vibrio vulnificus have anti-bacterial effects and their soluble epoxide hydrolase inhibitory activity [11]. However, due to the characteristics of multi-component, multi-pathway and multi-target synergistic effect, this complexity makes the substance basis of traditional Chinese medicine is not clear, the mechanism of action is not clear, the quality of traditional Chinese medicine and traditional Chinese medicine is difficult to control, and the evaluation system of efficacy and safety is lacking. It is difficult to carry out comprehensive and systematic research from the whole to the level of tissues, organs, cells and molecules. Network pharmacology through the construction of "disease-genetarget-drug" interaction network, docking drugs and multiple disease targets and network analysis, systematic and comprehensive observation of drug intervention and impact on the disease network [12], It is helpful to reveal the material basis and mechanism [13] of traditional Chinese medicine or compound.

\section{Materials And Methods}

Chinese medicine believes that the pathogenesis of early colorectal cancer (colorectal cancer) is dampness and heat toxin accumulation of large intestine, qi and blood stasis is not smooth, its treatment principles should be to clear heat and dampness, blood stasis and detoxification, commonly used prescriptions such as Huaijiao Diyu decoction, Baeweng decoction, Xuefu Zhuyu decoction, etc. As shown in Fig.1.

\subsection{Collection of active compounds and targets}


Through the TCMSP database (http://tcmspw.com/tcmsp.php), Search "Sophora japonica "," Ulmus pumila "," Fructus Aurantii "," Scutellaria baicalensis "," Schizonepeta "," Gardenia "," Radix Paeoniae Alba" seven herbs. Oral bioavailability (oral bioavailability,) in pharmacokinetic (ADME) parameters was used in this study $\mathrm{OB}$ and drug-like properties (drug-likeness,); and $\mathrm{DL}$ ) as a standard, $30 \% \mathrm{OB} \geq \mathrm{set}, \mathrm{DL} \geq 0.18$ to screen active compounds and predict target [14] of active compounds in Sophora flavescens decoction. The Uniprot database (https://www.uniprot.org/) is used to standardize the target name.

\subsection{Collection of CRC targets of active compounds in Sophora japonica L. Decoction}

Through the G enecards database (https://www.g enecards .org/) to "Colorectal cancer" as the keyword retrieval CRC action target [16]. The related targets of active compounds and CRC targets in Huaijiao Diyu decoction were mapped to obtain the intersection targets, and the $\mathrm{R}$ package VennDiagram was used to draw the Wayne map [17] of Huaijiao Diyu decoction and CRC related targets.

\subsection{Traditional Chinese Medicine - Active Compounds - Construction of CRC- Target Network}

The Chinese medicine, active compound, target and disease of Huaijiao Diyu decoction were introduced into the CRC Cytoscape 3.8.0 software to construct the "Chinese medicine-active compound- CRC- target" network. Analysis of topology parameters of network nodes using Network Analysis plug-ins, The core active compounds and core target [18] of Huaijiao Diyu decoction were screened by Degree value.

\subsection{Building protein interaction networks}

Using the string database (https://string-db.org/) to obtain target interaction [19], Homo sapiens", by selecting species Check "Hidden Network Interrupt Node hide disconnected nodes in the network", And set the "minimum required interaction score minimum required interaction score" $>0.9$ to get the protein interaction network diagram and the core genes of the network, According to the number of connections with adjacent genes, the top 30 core genes histogram was obtained by R software. Import data cytoscape database to draw protein interaction network. Analysis of topology parameters of network nodes using Network Analysis plug-ins, According to the Degree value, the Sophora japonica decoction was screened.

\subsection{Analysis of biological functions and pathways}

ClusterProfiler is a software package for enrichment clustering and visualization of gene sets in $\mathrm{R}$ languages. Using the R language (version 4.0.3) to call the clusterProfiler package to analyze the $\mathrm{GO}$ molecular function and KEGG pathway enrichment of the target, Among them, the screening condition is that the $P$ value is less than 0.05 . The histogram and bubble chart [20] of GO function and KEGG path are drawn by using $\mathrm{R}$ package.

\subsection{Molecular docking analysis of core active compounds with core protein receptors}

The core active compounds of the first 4 and the core protein receptors of the first 4 were selected from the interaction network of the CRC targets treated Degree Huaijiao Diyu decoction. The structure of target 
protein and core components are downloaded from PDB website (http://www.rcsb.org/) and pubchem website (https://pubchem.ncbi.nlm.nih.gov/) respectively, Using Pymol software to remove water and raw PDB, Autodock Tolls software for targeting protein receptor molecules with polar hydrogen, molecular docking through Autodock Vina and Python scripts. The lower the binding energy, the better the affinity between the receptor and the ligand, This study selected the binding energy $\leq-5.0 \mathrm{~kJ} / \mathrm{mol}$ as the screening basis for the effective binding of compounds and targets.

\section{Results}

\subsection{Screening results of active compounds and targets in Sophora japonica}

Enter the drug name in the TCMSP database, And according to $O B \geq 30, D L \geq$ the condition of 0.18 , the active components of the drug were obtained. There were 13 active compounds of E. officinalis, Six active compounds, Fructus Aurantii active compounds 5, There are 36 active compounds in Scutellaria baicalensis, There are 11 active compounds, Gardenia 5 active compounds, Radix Paeoniae Alba active compounds 13 , A total of 64 active compounds were obtained after mapping, A total of 247 corresponding targets.

\subsection{Results of intersection of active compounds in the treatment CRC action targets of Sophora japonica}

The $858 \mathrm{CRC}$ related potential targets were retrieved in the $\mathrm{G}$ enecards database, and 114 intersection targets were obtained by mapping them with 247 targets corresponding to Huaijiao Diyu decoction, as shown in Fig.2.

\subsection{Screening results of targets for the treatment of CRC by active compounds in Sophora flavescens decoction}

To construct the network map of Chinese medicine - active compound - CRC- target by introducing CRC Chinese medicine, active compound, target and disease into the software, The network diagram consists of 172 nodes and 491 edges, Common goals and diseases containing 58 ingredients and 114 drugs. 114 common targets are considered as potential targets for the treatment of CRC by huajiao diyu decoction (Fig.3). degree represents the total number of routes that other nodes in the network connect to that node. The higher the value, The more important the corresponding component or target is. After analysis using the circuit network analyzer plug-in in the Cytoscape software, Tables 1 and 2 list 10 major active components and 10 major targets, respectively. Table 1 shows, The highest degree component is PTGS2(degree 48).

\subsection{Building protein interaction networks}

Use the string database (https://string-db.org/) to obtain target interaction relationships, Homo sapiens", by selecting species Check "Hidden Network Interrupt Node hide disconnected nodes in the network", And set the "minimum required interaction score minimum required interaction score" $>0.9$ to get the protein interaction network diagram and the core genes of the network (Fig.4), According to the number of 
connections with adjacent genes, the top 30 core genes were obtained by R software (Fig.5). Import data cytoscape database to draw protein interaction network. Network Analysis plug-in analysis of the network diagram found, sorted by Degree values from high to low, AKT1, is the top 10 MAPK1, MAPK3, HSP90AA1, JUN, MAPK14, ESR1, CCND1, RB1, IL6( Fig.6).

\subsection{Analysis of biological functions and pathways}

GO function analysis shows, 114 intersection targets predicted 145 enrichment results (pvalueCutoff $<0.05$, qvalueCutoff $<0.05$ ). DNA-binding transcription factor binding, included RNA polymerase II-specific DNA-binding transcription factor binding, ubiquitin-like protein ligase binding and transcription coactivator binding, Based on the results of screening the top 20 of the P values, See Fig.7, Fig.8.

KEGG enrichment analysis of Sophora flavescens decoction $(p<0.05)$, The results show that 164 pathways were obtained, PI3K-Akt signaling pathway, MAPK signaling pathway and TNF signaling pathway were the representatives.. The top 20pathways in KEGG enrichment analysis was demonstrated according to $\mathrm{P}$ value (Fig.9, Fig.10) Furthermore, they were mainly involved in antiviral, immunomodulatory and anti-inflammatory effects according to the function of these top 20pathways (Table 3). Hepatitis B $\mathbb{X I D}$ hsa05161) is the clearest pathway,see Fig.11.

\subsection{Molecular docking}

Molecular docking results show, There were 12 pairs of quercetin, luteolin, kaempferol, baicalin and other four core components < the Vina score of 5.0 , indicating that the binding ability of PTGS1ロPTGS2ロAR and core components is strong, The Vina value (binding energy) of the interaction between PTGS1 protein and core components is the best, $\mathrm{kcal} / \mathrm{mol},-7.9$ The specific docking results are shown in Table 4. Combined with Fig.12, quercetin form hydrogen bonds ARG376ロGLN374 the amino acid residues of the PTGS2 protein, luteolin small molecules form hydrogen bonds THR206 the amino acid residues of the PTGS1 protein, wogonin small molecules form hydrogen bonds LYS93םTYR92 the amino acid residues of the AR protein.

\section{Discussion}

$\mathrm{CRC}$ is a common malignant tumor of digestive system, which can seriously affect the quality of life of patients. Huaijiao Diyu decoction has the function of reducing poison and increasing efficiency in the treatment of $\mathrm{CRC}$, but its specific molecular mechanism and pharmacodynamic substance have not been fully elucidated. At present, network pharmacology and molecular docking technology have been applied to the screening of active compounds of traditional Chinese medicine and the docking of key targets on a large scale, which provides a possibility for the preliminary exploration of the action law of Huaijiao Diyu decoction in the treatment of CRC.

Analysis of active ingredients active ingredient analysis 
Among the active ingredients, key ingredients with higher degree in-cludes quercetin,luteolin,kaempferol and wogonin. these four components belong to flavonoids, flavonoid phytoestrogens have been suggested to be associated with reduced risk of colorectal cancer [21].A recent research showed that the combination of quercetin and exercise training exerts potent anti-tumour and anti-depressive effects through suppression of inflammation and upregulation of the BDNF/TrK $\beta / \beta$-catenin axis in the prefrontal cortex in CRC treatment [22]. The combination of quercetin (Q) and alantolactone $(A)$ is capable of reactive activity of immunity by education ICD, causing cell toxicity and modulating the immunesuppressive tumor microenvironment [23]. Jiamei Qi [24]found that alternating the consumption of $\beta$ glucan and quercetin alleviated colon damage and reduced the mortality rate in CRC mice.During colon cancer genesis,luteolin known to reduce oxidative stress thereby protects the cell to undergo damage in vivo.Wnt/ $\beta$-catenin signaling,deregulated during neoplastic development,is modified by luteolin [25]. Thermosensitive in situ gel containing luteolin micelles has shown strong ability to promote tumor apoptosis, suppress tumor proliferation and block tumor angiogenesis [26]. luteolin uppresses colorectal cancer cell metastasis via regulation of the miR 384/pleiotrophin axis [27]. Kaempferoll has a synergistic effect with 5FU by inhibiting cell proliferation and inducing apoptosis in colorectal cancer cells via suppression of TS or attenuation of $p$ Akt activation. The combination of kaempferoll and 5FU may be used as an effective therapeutic strategy for colorectal cancer [28]. Reactive Oxygen Species and p53Mediated Activation of p38and Caspases is Critically Involved in Kaempferol Induced Apoptosis i n Colorectal Cancer Cells [29]. Wogonin could inhibit the proliferation of SW480cells through Wnt/ $\beta$-catenin pathway [30]. Wogonin exerts growth inhibitory effects on the SW48colorectal cancer cells by autophagic and apoptotic cell death,wogonin could also inhibit the PI3K/AKT and STAT3signal transduction pathways [31]. Wogonin not only reduced tumor multiplicity,preserved colon length to normal but also didn't induce side effects on various organs [32].

Analysis of potential targets Potential Target Analysis

The target with the highest degree was PTGS2. The following targets were PTGS1, HSP90AA1, AR in the network of ingredient-target-disease. prostaglandin endoperoxide synthase (PTGS), also known as cyclooxygenase (COX), which include inducible PTGS2(cox-2) and constitutive PTGS1(cox-1). HCT116 and HT29 could induce depolarization of granulocyte membrane potential after silencing by COX-1 gene, inhibiting adenosine triphosphate (ATP) production, Increasing the content of reactive oxygen species, leading to apoptotic [33] in cancer cells. The association of tumour PTGS2(COX-2) expression with colour cancer quality is strong in BRAF-mutated tonours than in BRAF-wild-type tonours [34]. Liu J and other [35] found Hsp90aa1 and dab2ip involved in the occurrence and metastasis mechanism of colorectal cancer. Heat shot protein 90(HSP90) AA1 functions as an onco-protein to regulate the assembly, manipulation, folding and degradation of its client proteins, including c-MYC and Weidong Shi's data revealed an unknown FBXL6-HSP90AA1-C-MYC axis which might contribute to the oncogenesis of HCC, and we propose that inhibition of FBXL6might represent an effective therapeutic strategy for HCC treatment [36]. Repression of $\beta 2$-AR but not $\beta 1$-AR signaling selectively suppressed cell viability, induced G1-phase cell cycle arrest, caused both intrinsic and extrinsic pathways-mediated apoptosis of specific CRC cells and inhibited CRC-xenograft growth in vivo [37]. 
Analysis of PPI network

PPI network analysis

The target protein levels of AKT1,MAPK1,MAPK3,HSP90AA1 and JUN were excessively expressed in PPI network. This result indicated the series of targets of Huaijiao Diyu decoction treating CRC.AKT, also known as protein kinase $B(P K B)$, is a downstream effector of the PI3K and is directly activated by it (PI3K Sator). Copanlisib promotes growth inhibition and apoptosis by modulating the AKT/FoxO3a/PUMA axis in colorectal cancer [38].A novel coordination complex of platinum (PT) leads cell death in colony cancer by after reducing balance and modelling MAPK pathway [39]. ROS/JNK/c-Jun axis is involved in oridonin-induced caspase-dependent apoptosis in human colorectal cancer cells [40].

Analysis of signaling pathways Signal Pathway Analysis

The results of GO molecular functional clustering of 114 potential target genes in clusterProfiler package showed that the top 30 enrichment results involve many molecular functions, such as cell signal transduction, cell metabolism, transcription and redox, as well as antiviral, immunomodulatory and antiinflammatory effects.KEGG pathway enrichment suggests, Possible CRC and PI3K-Akt signaling pathway, of Tongxie prescription MAPK signaling pathway and TNF signaling pathway closely related. Among them, Most CRC patients with abnormal PI3K/AKT signaling pathway and PI3K/Akt signal inhibitors are considered to be effective CRC therapeutic agents. PI3K/AKT signaling pathways can regulate the transcriptional expression of key miRNA, To enhance the [42] of resistance to proliferation and migration of CRC cells.Huijie Zhang and other [43] studies have found that regulating the activity of mapk signaling pathway can improve the depression behavior and survival state of the rat depression model. The RAS/MAPK axis regulates cancer cell proliferation,apoptosis,inflammation,migration,and metastasis [44]. Jobin and colleagues [45]found that targeting inflammation with TNF therapy has a preventative effect on carcinogenic activity of the microbiota in mouse models of colitis-associated colorectal cancer. molecular docking indicates that the core components of Huaijiao Diyu decoction interact strongly with

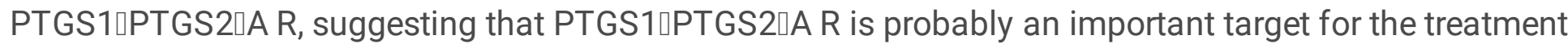
of Huaijiao Diyu decoction.

\section{Conclusion}

This study used network pharmacology, molecular docking and other means to obtain 58 active compounds and 114 targets of CRC treated by Huaijiao Diyu decoction, and demonstrated the molecular relationship and the signal pathway covered by the GO function enrichment analysis, the KEGG pathway enrichment analysis, the construction of the CRC- target network and the key target verification.The results showed that there was a synergistic effect between traditional Chinese medicine, and showed that Huaijiao Diyu decoction could play a pharmacological role at the molecular level through multicomponent, multi-target and multi-way, mainly involving many molecular functions such as cell signal transduction, cell metabolism, transcription and redox, as well as antiviral, immunomodulatory and antiinflammatory effects. The overall understanding of the treatment CRC of Huaijiao Diyu decoction from an 
intuitive point of view provides an important reference for the further study of the intervention CRC of Huaijiao Diyu decoction, which presents some advantages compared with the traditional pharmacological research. Limited by this study, only considering the quantitative relationship between traditional Chinese medicine, active compounds and targets, but not taking into account the effective intensity factors of active compounds and targets, the mechanism of action of drugs in vivo needs to be verified by further experiments. Therefore, more research methods should be carried out in the future to further reveal the mechanism of common prescriptions and their derived compounds in the treatment of colorectal cancer.

\section{Abbreviation}

CRC: Colorectal Cancel; TCM: Traditional Chinese medicine; TCMSP: Traditional Chinese Medicine Systems Pharmacology; OB: Oral bioavailability; DL: Drug likeness; PPI: Protein-protein interaction; STRING: Search Tool for the Retrieval of Interacting Genes/Proteins database; GO: Gene Ontology; KEGG: Kyoto Encyclopedia of Genes and Genomes; PDB: Protein Data Bank.

\section{Declarations}

\section{Ethics approval and consent to participate}

Not applicable

\section{Consent for publication}

Not applicable

\section{Availability of data and materials}

Not applicable

\section{Competing interests}

The authors declare that they have no competing interests

\section{Funding}

Not applicable

\section{Authors' contributions}

Xinyao Shu-SXY

Yunwu Zhang- ZYW

Yujia Wang-WYJ 
Jing Wu- WJ

Xingyu Chen- CXY

Ruitong Xu- XRT

Xingting Liu- LXT

Jingsong Luo- LJS

Tingting Deng- DTT

ZYW, DTT contributed to the conception of the study;

SXY performed the experiment;

SXY, WYJ contributed significantly to analysis and manuscript preparation;

ZYW, SXY, WJ performed the data analyses and wrote the manuscript;

CXY, XRT, LXT, LJS helped perform the analysis with constructive discussions.

\section{Acknowledgements}

Not applicable

\section{References}

1. Yao,Y.,et al.,Luteolin suppresses colorectal cancer cell metastasis via regulationof the miR -384/pleiotrophin axis. Oncology Reports,2019.42(1).

2. Di Leo M, Z.R.,Puzzono M, Ditonno I, Mannucci A, Antoci G, Russo Raucci A, Patricelli MG, Elmore U, Tamburini AM, Albarello L, Azzolini F, Bonura GF, Esposito D, Fanti L, Notaristefano C, Viale E, Perea J, Testoni PA, Rosati R, Cavestro GM, Risk factors and clinical characteristics of early-onset colorectal cancer vs.late-onset colorectal cancer囚a case-case study.European journal of gastroenterology \&hepatology, 2020.

3. Bray,F.,et al.,Global cancer statistics 2018DGLOBOCAN estimates of incidence and mortality

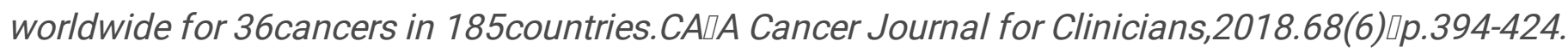

4. Yuji,M.,et al.,Precision medicine for adjuvant chemotherapy of resected colorectal cancer.Annals of Gastroenterological Surgery,2020.4(6).

5. Shi-Yun,S.and L.Qi,Research progress in the treatment of colorectal cancer in classical prescriptions. TMR Cancer,2019.2(3).

6. Yuli,W.,et al.,The Effect of Long-Term Traditional Chinese Medicine Treatment on Survival Time of Colorectal Cancer Based on propensity Score Matching $\square$ A Retrospective Cohort Study.Evidence-based complementary and alternative medicine \eCAM,2020.2020. 
7. Shao,C.,et al.,Effect of Chinese Herbal Medicine on the Survival of Colorectal Cancer Patients With Liver-Limited Metastases $\square$ A Retrospective Cohort Study,2008to 2017.Integrative Cancer Therapies,2019.18.

8. Hyun,C.Y.and K.Hye-Joo,Fructus sophorae attenuates secretion of proinflammatory mediators and cytokines through the modulation of NF-KB and MAPK signaling pathways in LPS-stimulated RAW 264.7macrophages. General physiology and biophysics,2016.35(3).

9. Han,H.M.,et al.,Protective effects of Fructus sophorae extract on collagen-induced arthritisin BALB/C mice.Experimental and Therapeutic Medicine,2017.13(1).

10. Hong,Z.,et al.,Positive effects of platelet-rich plasma (PRP) and a Sanguisorba officialis polysyaccharide on the protection and differentiation of anteriorcruciate ligament (ACL) fibroblasts in vitro. Pharmacological biology ,2020.58(1).

11. Dong,S.X.,et al.,Anti-bacterial effects of components from Sanguisorba officinalis L.on Vibrio vulnificus and their soluble epoxide hydrolase inhibitory activity.Natural product research,2019.33(23).

12. Bo,Z.,et al.,Matrine Is Identified as a Novel Macropinocytosis Inducer by a Network Target Approach. Frontiers in pharmacology,2018.9.

13. Runzhi,Z.,et al.,Network Pharmacology Databases for Traditional Chinese MedicinelReview and Assessment.Frontiers in pharmacology,2019.10.

14. Ru,J.,et al.,TCMSPIa database of systems pharmacology for drug discovery from herbal medicines.Journal of Cheminformatics,2014.6(1).

15. Wenjuan,Z.,et al.,Systems Pharmacology for Investigation of the Mechanisms of Action of Traditional Chinese Medicine in Drug Discovery. Frontiers in pharmacology,2019.10.

16. Yunxia,W.,et al.,Therapeutic target database 2020■enriched resource for facilitating research and early development of targeted therapeutics. Nucleic acids research,2020.48(D1).

17. Chen,H.and P.C.Boutros, VennDiagram $\square$ a package for the generation of highly-customizable Venn and Euler diagrams in R.BMC Bioinformatics,2011.12(1).

18. T,D.N.,et al.,Cytoscape StringApp $\square$ Network Analysis and Visualization of Proteomics Data.Journal of proteome research,2019.18(2).

19. Szklarczyk,D.,et al.,The STRING database in 2017ロquality-controlled protein-protein association networks,made broadly accessible.Nucleic Acids Research,2017.45(D1).

20. Walter,W.,F.t.Saanchez - Cabo,and M.Ricote,GOplotDan R package for visually combining expression data with functional analysis.Bioinformatics,2015.31(17).

21. Ruijingfang,J.,et al.,Serum Concentration of Genistein,Luteolin and Colorectal Cancer Prognosis.Nutrients,2019.11(3).

22. Sadighparvar,S.,et al.,Combination of quercetin and exercise training attenuates depression in rats with 1,2-dimethylhydrazine-induced colorectal cancer $\square$ Possible involvement of inflammation and BDNF signalling. Experimental Physiology,2020.105(9). 
23. Jing,Z.,et al.,Nanoformulated Codelivery of Quercetin and Alantolactone Promotes an Antitumor Response through Synergistic Immunogenic Cell Death for Microsatellite-Stable Colorectal Cancer.ACS nano,2019.13(11).

24. Qi,J.,et al.,Alternating consumption of $\beta$-glucan and quercetin reduces mortality in mice wi th colorectal cancer.Food Science \&Nutrition,2019.7(10).

25. Kumar,P.A. and E.N.Mohd, Luteolin, a bioflavonoid inhibits colorectal cancer through modulation of multiple signaling pathways $\square$ a review.Asian Pacific journal of cancer prevention $\square A P J C P, 2014.15(14)$.

26. Yuzhu,H.,et al.,Thermosensitive In Situ Gel Containing Luteolin Micelles is a Promising Efficient Agent for Colorectal Cancer Peritoneal Metastasis Treatment. Journal of biomedical nanotechnology,2020.16(1).

27. Yuanyuan, Y.,et al.,Luteolin suppresses colorectal cancer cell metastasis via regulation of the miR -384/pleiotrophin axis. Oncology reports,2019.42(1).

28. Qiongyu,L.,et al.,Synergistic effect of kaempferol and 5-fluorouracil on the growth of colorectal cancer cells by regulating the PI3K/Akt signaling pathway.Molecular medicine reports,2019.20(1).

29. Jhin-Baek,C.,et al.,Reactive Oxygen Species and p53Mediated Activation of p38and Caspases is Critically Involved in Kaempferol Induced Apoptosis in Colorectal Cancer Cells.Journal of agricultural and food chemistry,2018.

30. Yan-Ping,L.,et al.,[Inhibitory effect of wogonin on human colorectal cancer cell SW480based on network pharmacology]. Zhongguo Zhong yao za zhi =Zhongguo zhongyao zazhi =China journal of Chinese materia medica,2020.45(8).

31. Hao, T.,et al.,A flavone,Wogonin from Scutellaria baicalensis inhibits the proliferation of human colorectal cancer cells by inducing of autophagy,apoptosis and G2/M cell cycle arrest via modulating the PISK/AKT and STAT3signalling pathways. Journal of B.U.ON: official journal of the Balkan Union of Oncology,2019.24(3).

32. Qian,F.,et al.,Prevention of Wogonin on Colorectal Cancer Tumorigenesis by Regulating p53Nuclear Translocation.Frontiers in pharmacology,2018.9.

33. Lei,D.,et al.,Downregulation of cyclooxygenase -1stimulates mitochondrial apoptosis through the NF -KB signaling pathway in colorectal cancer cells. Oncology reports,2019.41(1).

34. Kosumi,K.,et al.,Prognostic association of PTGS2(COX-2) over-expression ordering to BRAF mutation status in color cancer: Results from two constructive cases and CALGB 89803(Alliance) priority. European Journal of Cancer ,2019.111.

35. J., L.Bioinformatic Analysis and Mechanism Study of Expression Relationship between HSP90AA1and DAB2IP in Colorectal Cancer in 17th International Immunology Conference .2019. Beijing, China.

36. Shi,W.et al.,FBXL6governs c-MYC to promote hepatocellular carcinoma through ubiquitination and stabilization of HSP90AA1.Cell Communication and Signaling,2020.18(1).

37. Chin,C.C.,et al.,Selective $32-$ AR Blockage Suppresses Colorectal Cancer Growth Through Regulation of EGFR-Akt/ERK1/2Signaling,G1-Phase Arrest,and Apoptosis. Journal of Cellular 
Physiology,2016.231(2).

38. Ji,Y,et al.,Copanlisib promotes growth inhibition and apoptosis by modulating the AKT/Fox03a/PUMA axis in colorectal cancer.Cell Death \&Disease,2020.11(11).

39. Al-Khayal,K.,et al.,A novel coordination complex of platinum (PT) leads cell death in coloral cancer by after reducing balance and modelling MAPK pathway. BMC Cancer ,2020.20(1).

40. Di,Z.,et al.,ROS/JNK/C-Jun axis is involved in oridonin-induced caspase-dependent apoptosis in human colorectal cancer cells.Biochemical and Biophysical Research Communications,2019.513(3).

41. Kumar,P.A.,Potential targets for prevention of colorectal cancer $\square$ a focus on PI3K/Akt/mTOR and Wnt pathways.Asian Pacific journal of cancer prevention $\square A P J C P, 2013.14(4)$.

42. Soleimani,A.,et al.,Role of Regulatory Oncogenic or Tumor Suppressor miRNAs of PI3K/AKT Signaling Axis in the Pathogenesis of Colorectal Cancer.Current Pharmaceutical Design,2018.24(39).

43. Zhang H, C.M.,Liu Y, Dong X, Zhang C, Jiang H, Chen X, Paroxetine combined with fluorouracil plays a therapeutic role in mouse models of colorectal cancer with depression through inhibiting IL22expression to regulate the MAPK signaling pathway. Experimental and therapeutic medicine, 2020.20(6).

44. Soleimani,A.,et al., The potential role of regulatory microRNAs of RAS/MAPK signaling pathway in the pathogenesis of colorectal cancer.Journal of Cellular Biochemistry,2019.120(12).

45. Yang,Y.,et al.,Amending microbiota by targeting intestinal inflammation with TNF blockade attenuates development of colorectal cancer.Nature Cancer,2020.1(7).

\section{Tables}

Table 1 Core targets of the top 10 in the network of Degree CRC-targets

\begin{tabular}{|lllll|}
\hline Gene & Degree & TopologicalCoefficient & NeighborhoodConnectivity & Radiality \\
\hline PTGS2 & 48 & 0.078683036 & 9.8125 & 0.989747095 \\
\hline PTGS1 & 38 & 0.094976077 & 11.44736842 & 0.987924356 \\
\hline HSP90AA1 & 38 & 0.097054563 & 11.57894737 & 0.987772461 \\
\hline AR & 24 & 0.139823718 & 15.54166667 & 0.984886459 \\
\hline PRSS1 & 23 & 0.142140468 & 15.7826087 & 0.984734564 \\
\hline NOS2 & 21 & 0.200746965 & 11.23809524 & 0.976380345 \\
\hline ESR1 & 13 & 0.211538462 & 10.30769231 & 0.974101921 \\
\hline PPARG & 11 & 0.21991342 & 24.09090909 & 0.98306372 \\
\hline PGR & 10 & 0.136363636 & 5.5 & 0.971671603 \\
\hline CASP3 & 10 & 0.236792453 & 26.1 & 0.98306372 \\
\hline
\end{tabular}


(Notes囚PTGS2prostaglandin-endoperoxide synthase 2,) Annex PTGS1prostaglandin-endoperoxide synthase 1, HSP90AA1 heat shock protein 90alpha family class A member 1, AR androgen receptor, PRSS1serine protease 1, NOS2nitric oxide synthase 2, ESR1Estrogen Receptor 1, PPARG peroxisome proliferator activated receptor gamma, PGR progesterone receptor, CASP3caspase 3)

Table 2 Core Compounds with Top 10 Values in the Network of Degree CRC-Targets

\begin{tabular}{|llll|}
\hline MOL ID & Molecule Name & Degree & Source \\
\hline MOL000098 & quercetin & 77 & diyu,huaijiao,jingjie,zhizi \\
\hline MOL000006 & luteolin & 40 & jingjie \\
\hline MOL000422 & kaempferol & 28 & baishao,diyu,huaijiao,zhizi \\
\hline MOL000173 & wogonin & 27 & huangqin \\
\hline MOL002714 & baicalein & 23 & huaijiao,huangqin \\
\hline MOL005828 & nobiletin & 21 & zhike \\
\hline MOL001689 & acacetin & 18 & huangqin \\
\hline MOL004328 & naringenin & 18 & zhike \\
\hline MOL002928 & oroxylin & 12 & huangqin \\
\hline MOL002933 & 5,7,4'- Trihydroxy-8-methoxyflavone & 12 & huangqin \\
\hline
\end{tabular}

Table 3 Information of potential targets and signaling pathways 


\begin{tabular}{|llll|}
\hline ID & Description & pvalue & Count \\
\hline hsa04151 & Pl3K-Akt signaling pathway & $3.73 \mathrm{E}-20$ & 34 \\
\hline hsa05161 & Hepatitis B & $2.23 \mathrm{E}-30$ & 33 \\
\hline hsa05167 & Kaposi sarcoma-associated herpesvirus infection & $3.71 \mathrm{E}-25$ & 31 \\
\hline hsa05163 & Human cytomegalovirus infection & $9.96 \mathrm{E}-21$ & 29 \\
\hline hsa05166 & Human T-cell leukemia virus 1infection & $6.46 \mathrm{E}-20$ & 28 \\
\hline hsa05215 & Prostate cancer & $8.43 \mathrm{E}-29$ & 27 \\
\hline hsa05169 & Epstein-Barr virus infection & $1.01 \mathrm{E}-19$ & 27 \\
\hline hsa04210 & Apoptosis & $3.99 \mathrm{E}-23$ & 26 \\
\hline hsa05205 & Proteoglycans in cancer & $2.05 \mathrm{E}-18$ & 26 \\
\hline hsa05206 & MicroRNAs in cancer & $5.60 \mathrm{E}-14$ & 26 \\
\hline hsa05165 & Human papillomavirus infection & $2.64 \mathrm{E}-13$ & 26 \\
\hline hsa01522 & Endocrine resistance & $1.28 \mathrm{E}-25$ & 25 \\
\hline hsa05162 & Measles & $1.49 \mathrm{E}-21$ & 25 \\
\hline hsa05160 & Hepatitis C & $3.43 \mathrm{E}-20$ & 25 \\
\hline hsa05170 & Human immunodeficiency virus 1infection & $7.11 \mathrm{E}-16$ & 24 \\
\hline hsa04010 & MAPK signaling pathway & $1.11 \mathrm{E}-12$ & 24 \\
\hline hsa05022 & Pathways of neurodegeneration -multiple diseases & $2.22 \mathrm{E}-08$ & 24 \\
\hline hsa04933 & AGE-RAGE signaling pathway in diabetic complications & $1.84 \mathrm{E}-22$ & 23 \\
\hline hsa04218 & Cellular senescence & $7.86 \mathrm{E}-18$ & 23 \\
\hline hsa05225 & Hepatocellular carcinoma & $4.31 \mathrm{E}-17$ & 23 \\
\hline
\end{tabular}

Table 4 Binding energies of key active compounds with core targets

\begin{tabular}{|llllll|}
\hline Mol ID & Molecule Name & PTGS1 & PTGS2 & HSP90AA1 & AR \\
\hline MOL000098 & quercetin & -5.4 & -7.9 & -4.2 & -7.5 \\
\hline MOL000006 & luteolin & -6.3 & -7.9 & -4.2 & -7.3 \\
\hline MOL000422 & kaempfero & -5.4 & -7.9 & -4.2 & -7.1 \\
\hline MOL000173 & wogonin & -6.2 & -7.9 & -4.2 & -7.5 \\
\hline
\end{tabular}




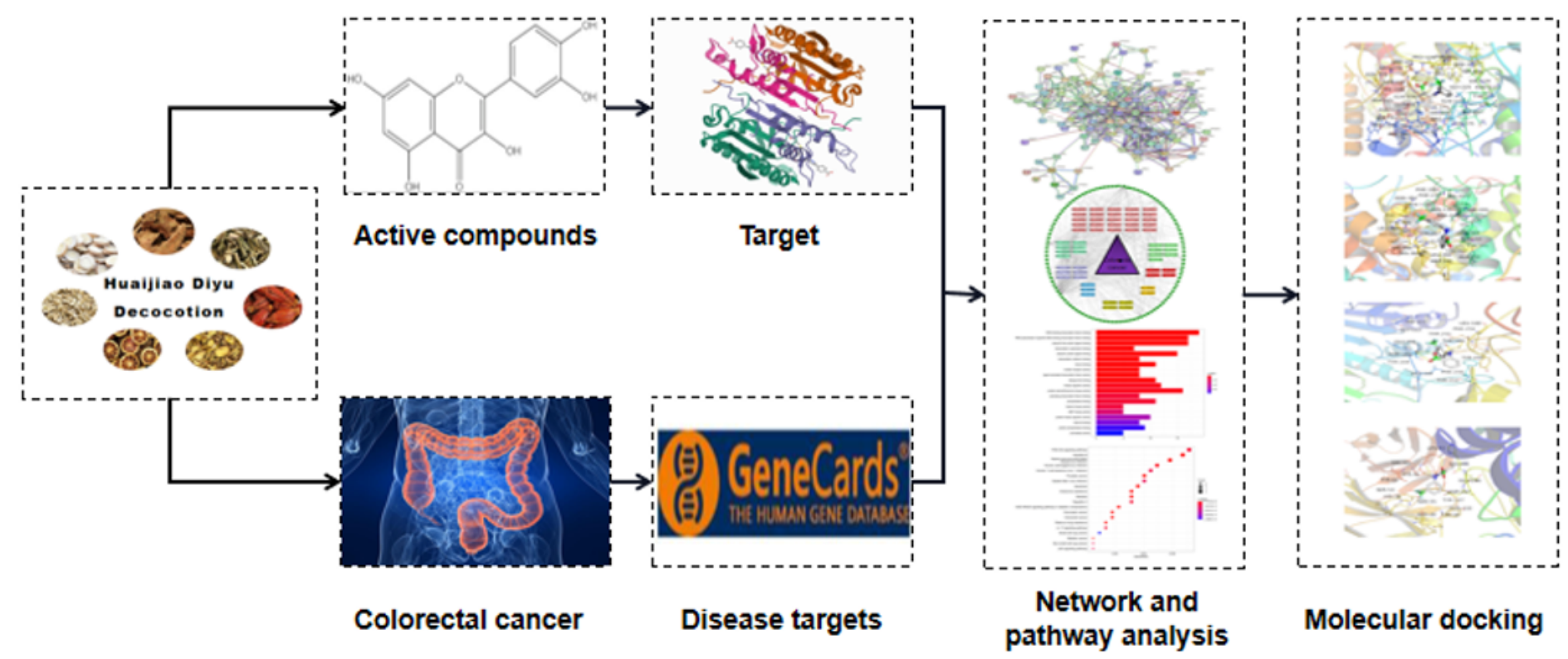

Figure 1

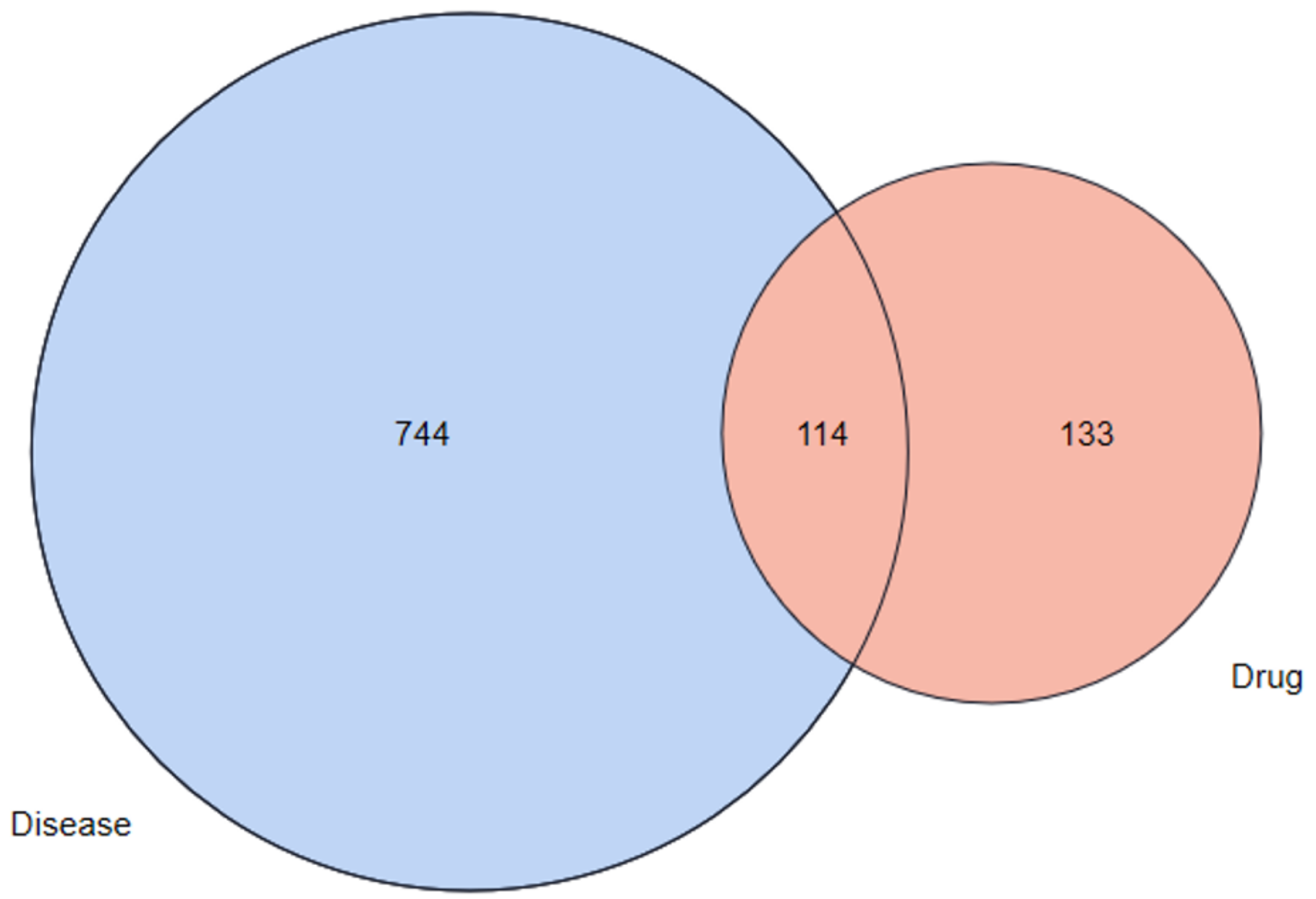




\section{Figure 2}

Venn diagram of Huaijiao Diyu decoction-CRC related targets (the large circle on the left represents the CRC related potential target, the small circle on the right represents the related potentiVenn diagram of Huaijiao Diyu decoction-CRC related targets (the large circle on the left represents the CRC related potential target, the small circle on the right represents the related potential target of Huaijiao Diyu decoction, and the middle represents the intersection target. )al target of Huaijiao Diyu decoction, and the middle represents the intersection target. )

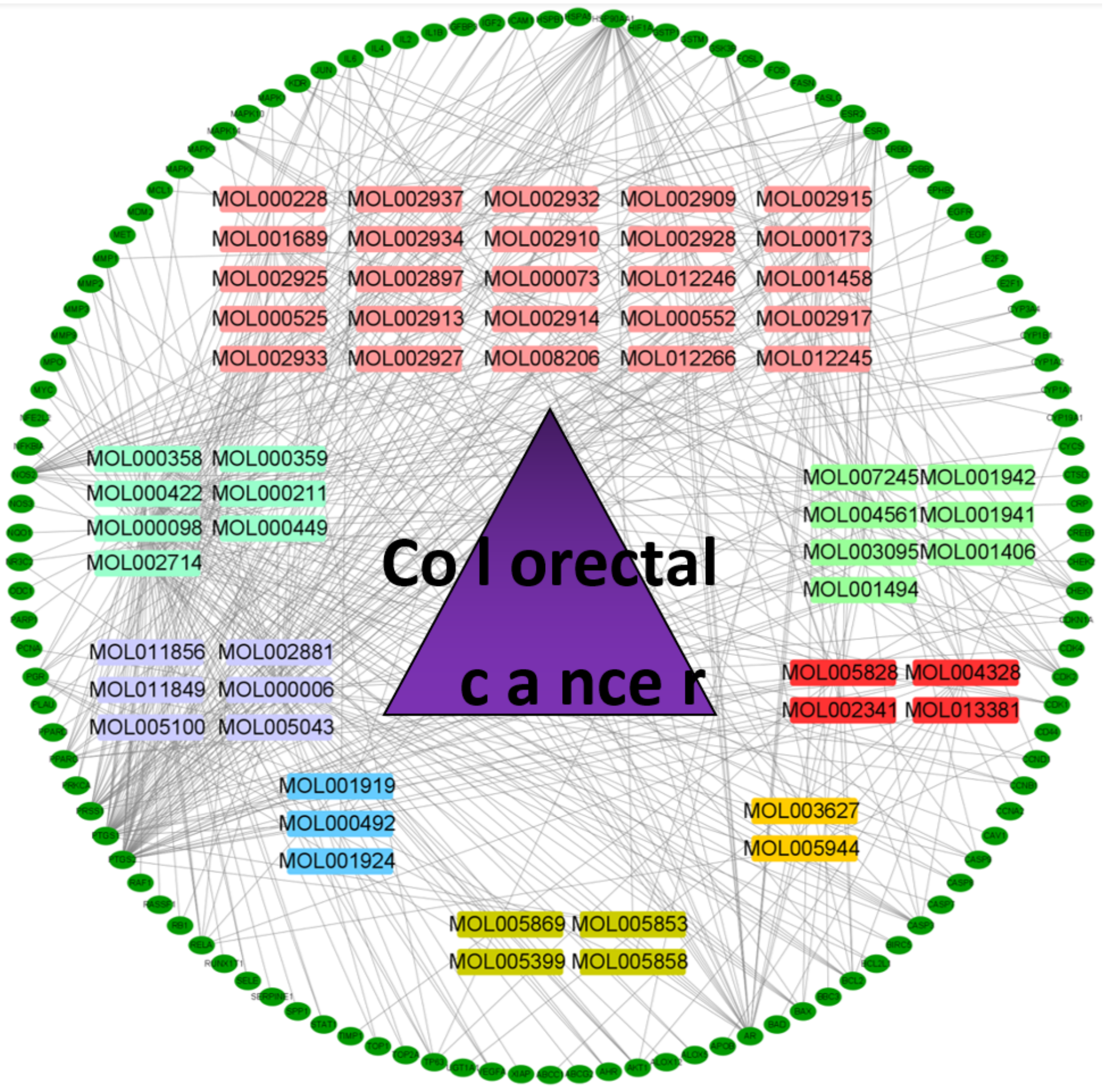

Figure 3 
The network of diagram of herbs-active compounds-targets (triangle represents $\mathrm{CRC}$, rectangle represents the corresponding active compounds of traditional Chinese medicine, oval represents the target of active compounds, light pink represents Scutellaria baicalensis and its corresponding active compounds, light blue indicates Schizonepeta mutiDrug, gray indicates Schizonepeta and its corresponding active compounds, dark yellow indicates Ulmus pumila and its corresponding active compounds, orange indicates Sophora japonica and its corresponding active compounds, red indicates Fructus Aurantii and its corresponding active compounds, light green indicates gardenia and its corresponding active compounds. ) 


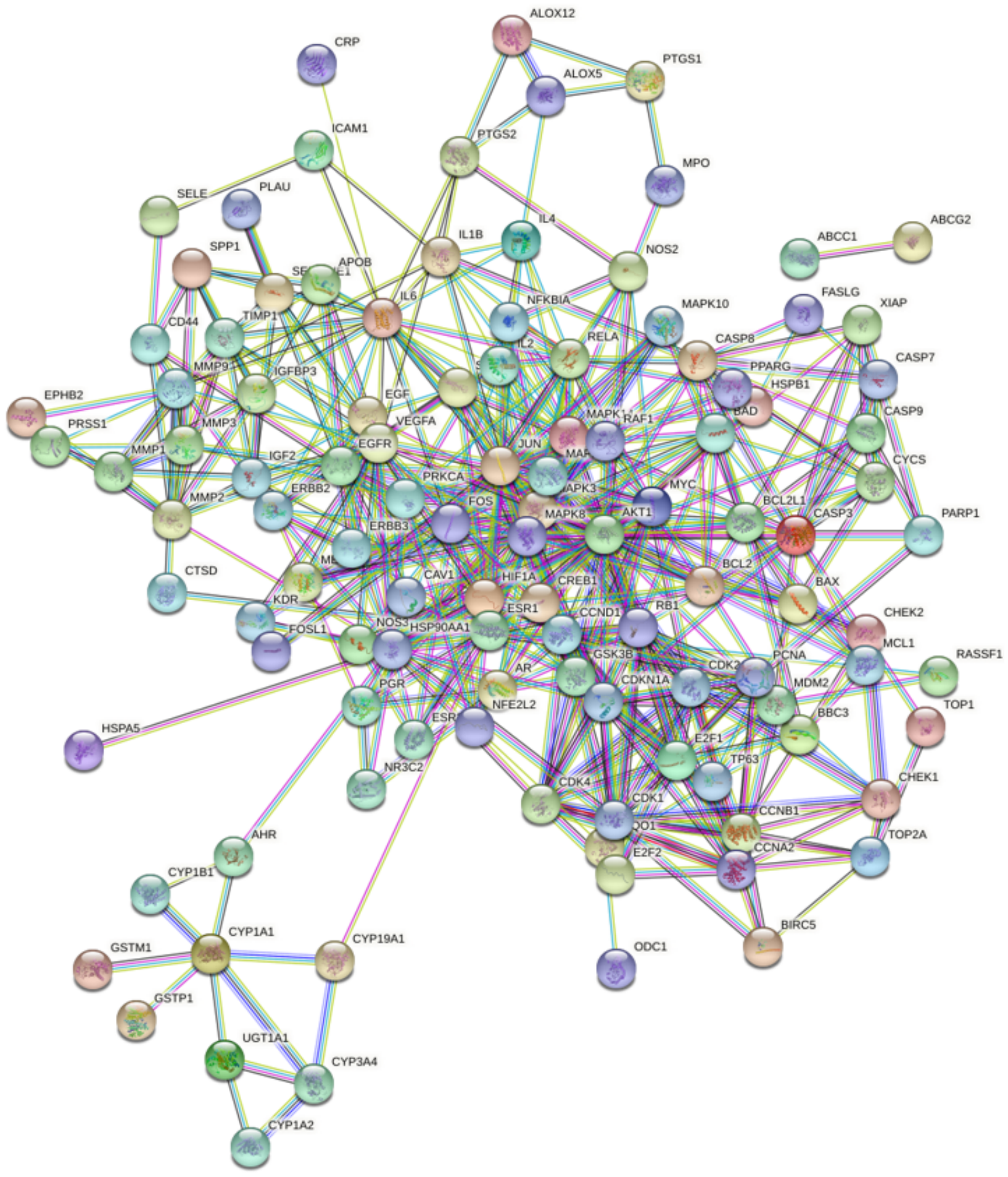

Figure 4

The network of protein to protein interaction (PPI) 


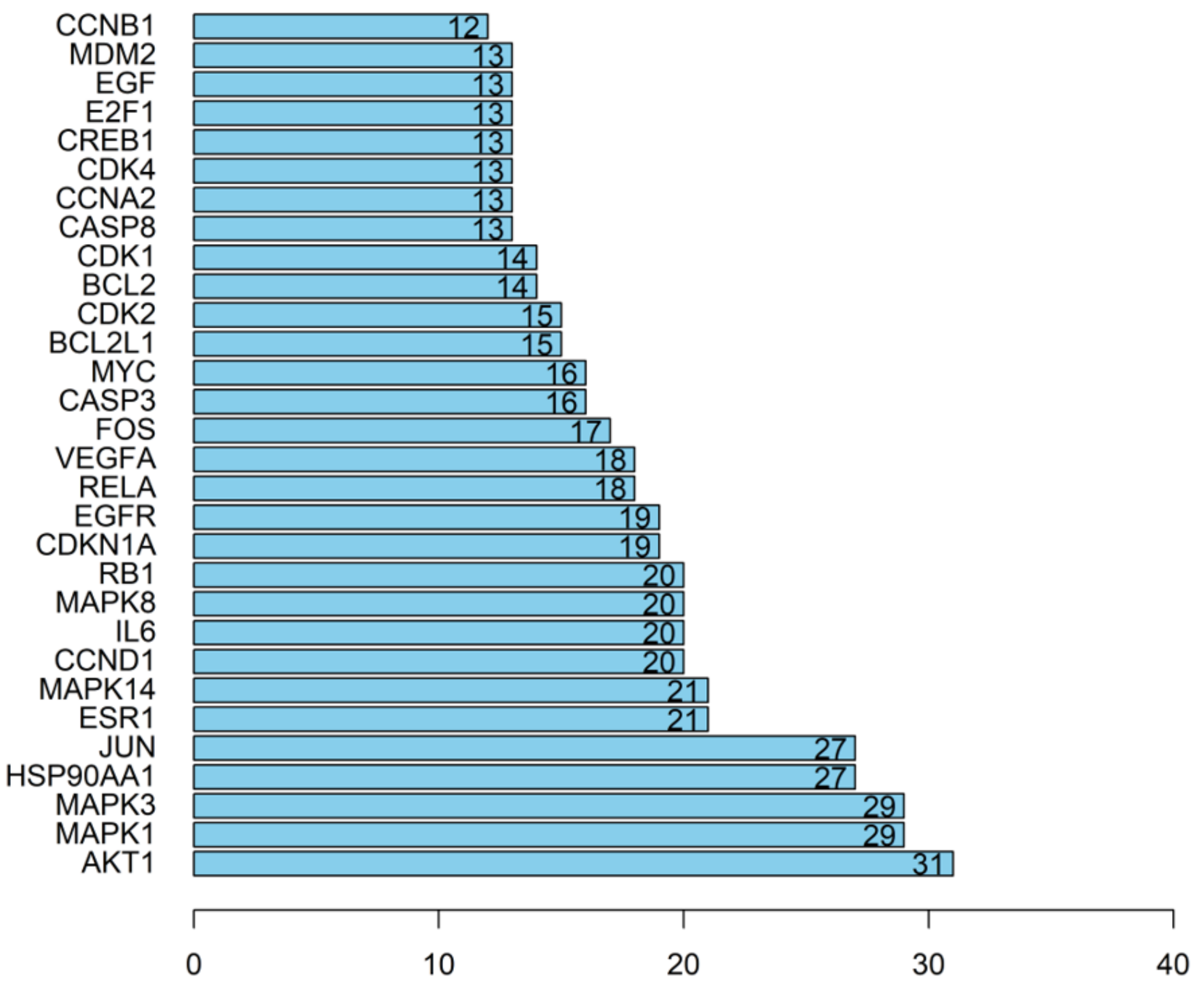

Figure 5

The top 30 core genes 


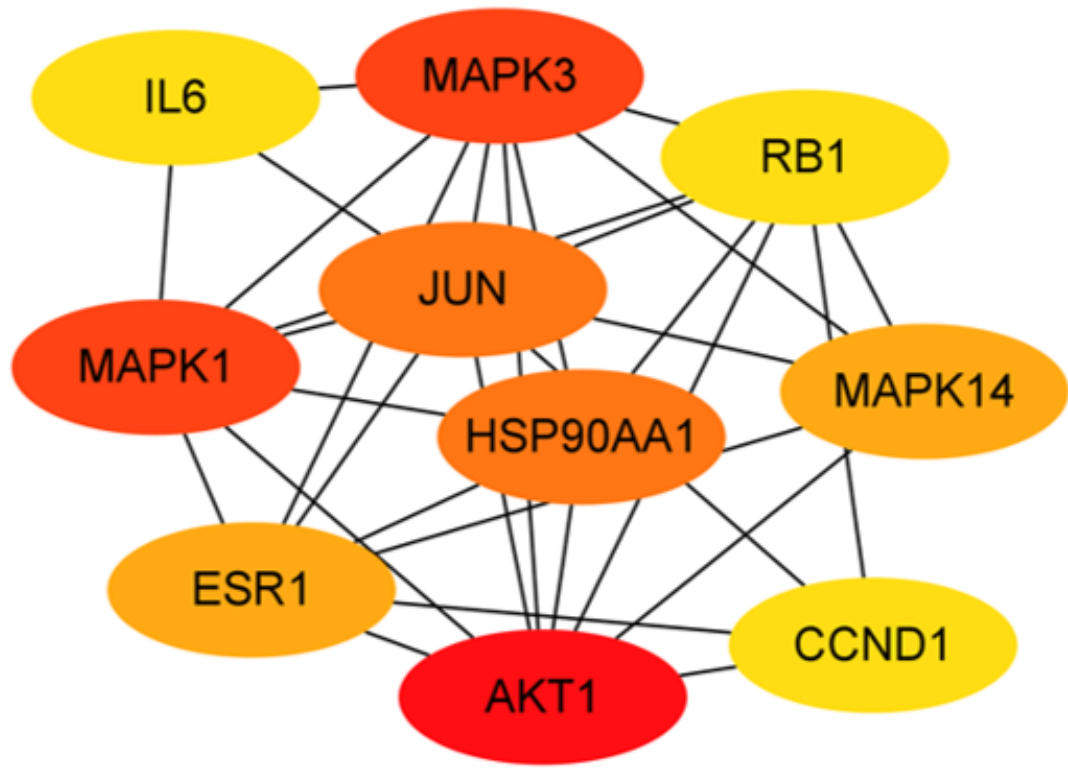

\begin{tabular}{|l|l|}
\hline Rank: & $\begin{array}{l}\text { Iode } \\
\text { AE T1 }\end{array}$ \\
\hline 1 & ILAPK 1 \\
\hline 2 & ILAPK 3 \\
\hline 2 & HSP90AA1 \\
\hline 4 & JUI \\
\hline 4 & ILAPK 14 \\
\hline 6 & ESR1 \\
\hline 6 & CCID 1 \\
\hline 8 & RB1 \\
\hline 8 & IL 6 \\
\hline 8 & \\
\hline
\end{tabular}

\section{Figure 6}

The core target of Degree top 10 in the network of protein interactions (AKT1,MAPK1, MAPK3, HSP90AA1, JUN, MAPK14, ESR1, CCND1, RB1, IL6)

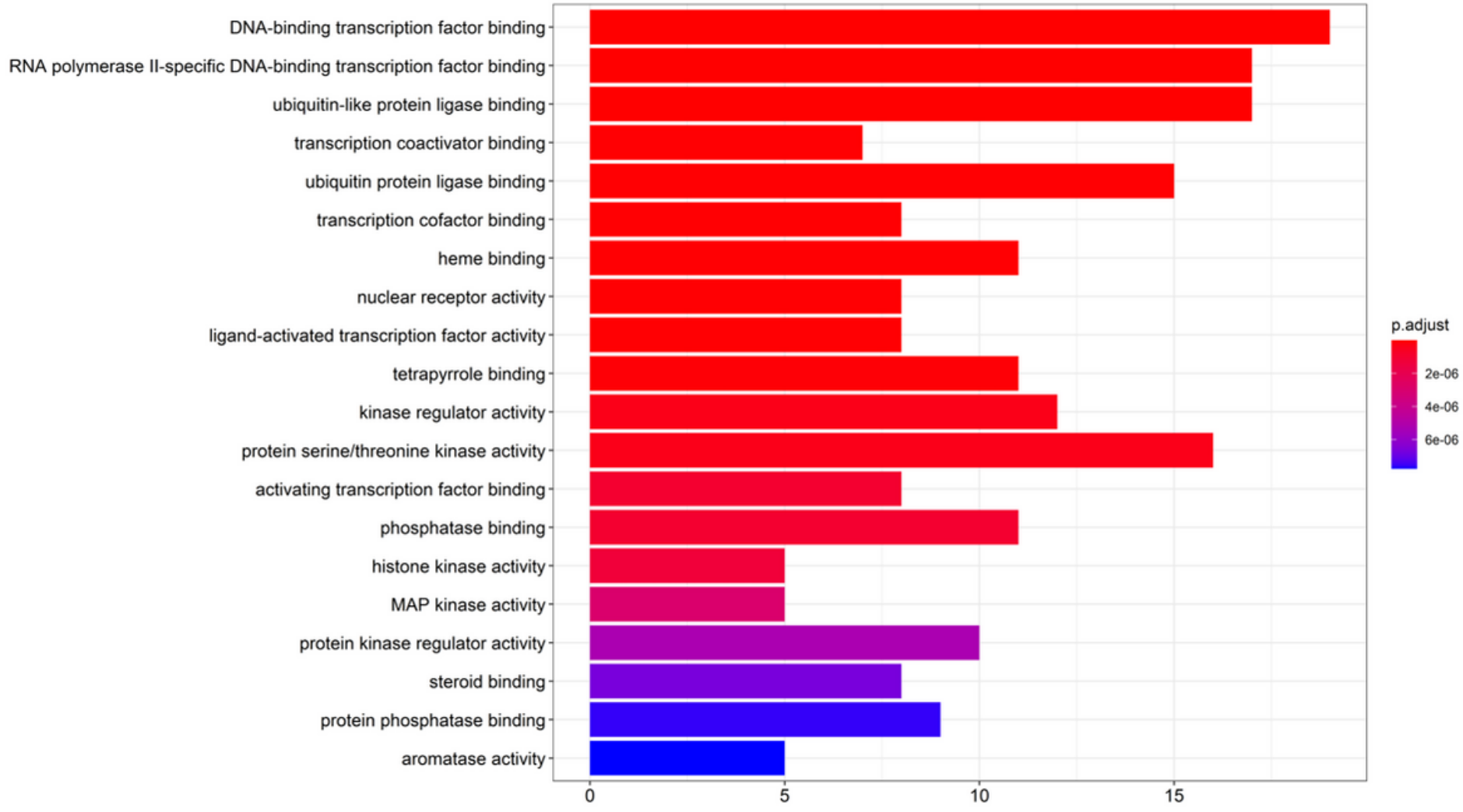


Figure 7

GO function histogram of the key targets of Huaijiao Diyu decoction in treatment of CRC

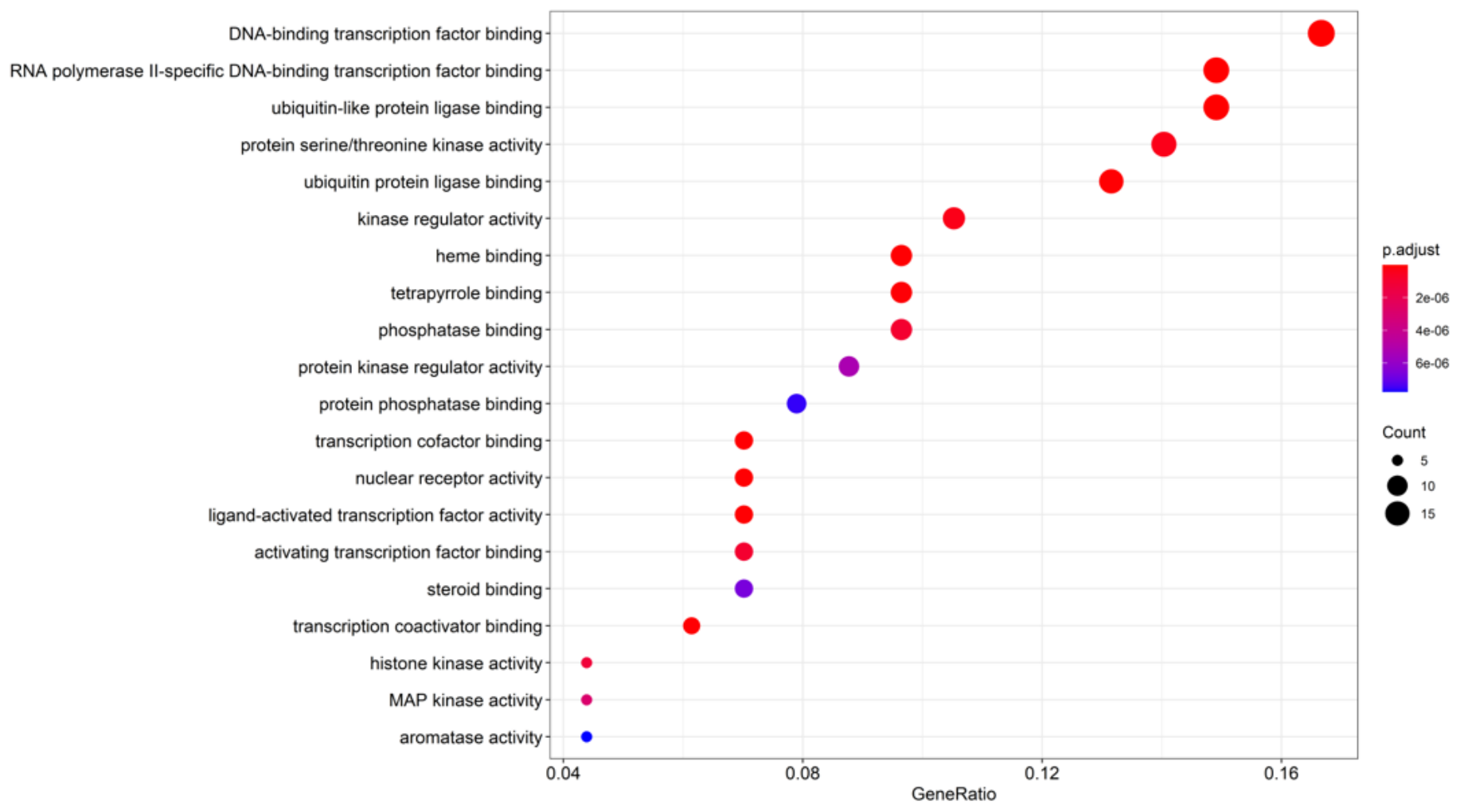

Figure 8

GO pathway enrichment bubble chart of the key targets of Huaijiao Diyu decoction in treatment of CRC 


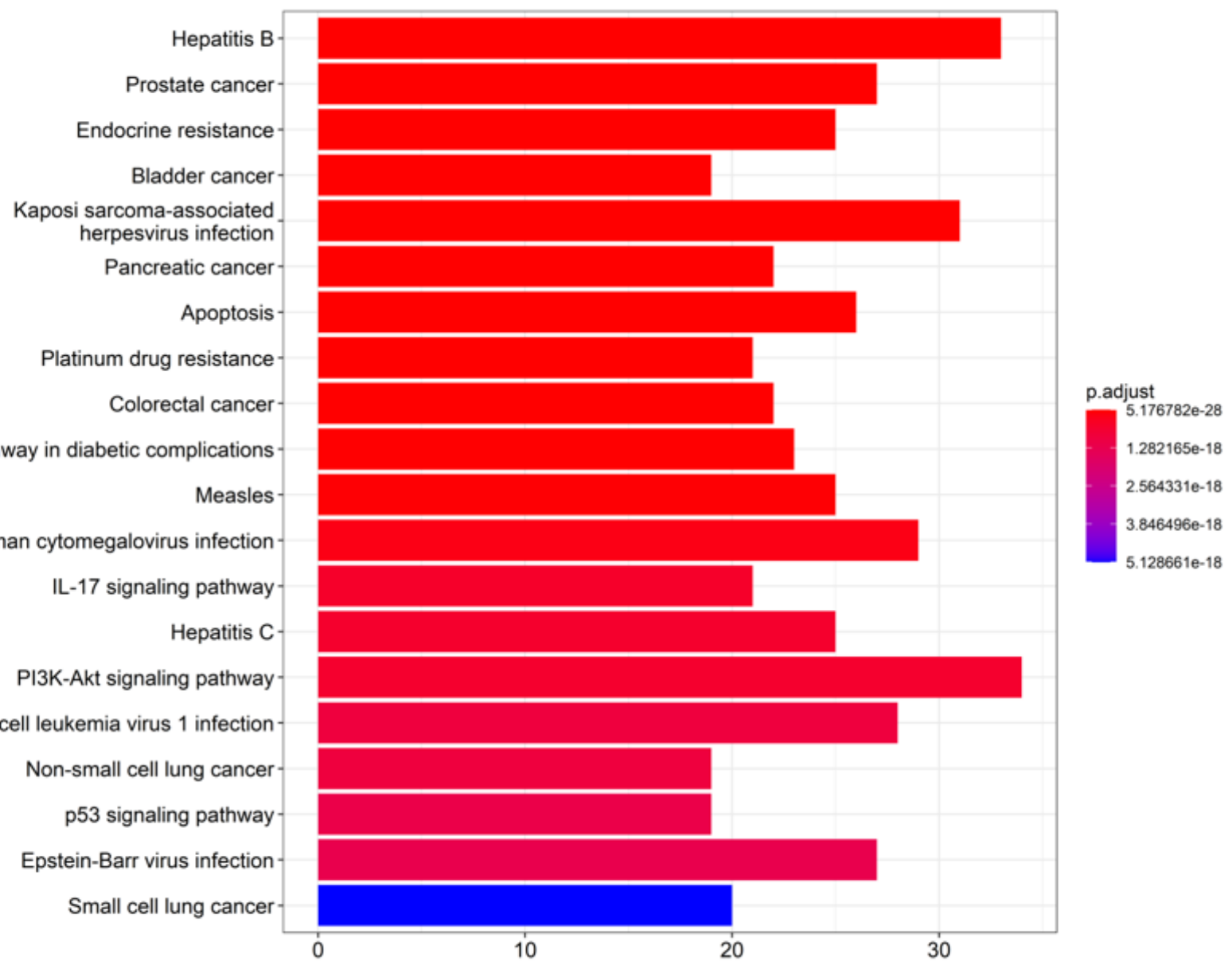

Figure 9

KEGG function histogram of the key targets of Huaijiao Diyu decoction in treatment of CRC 


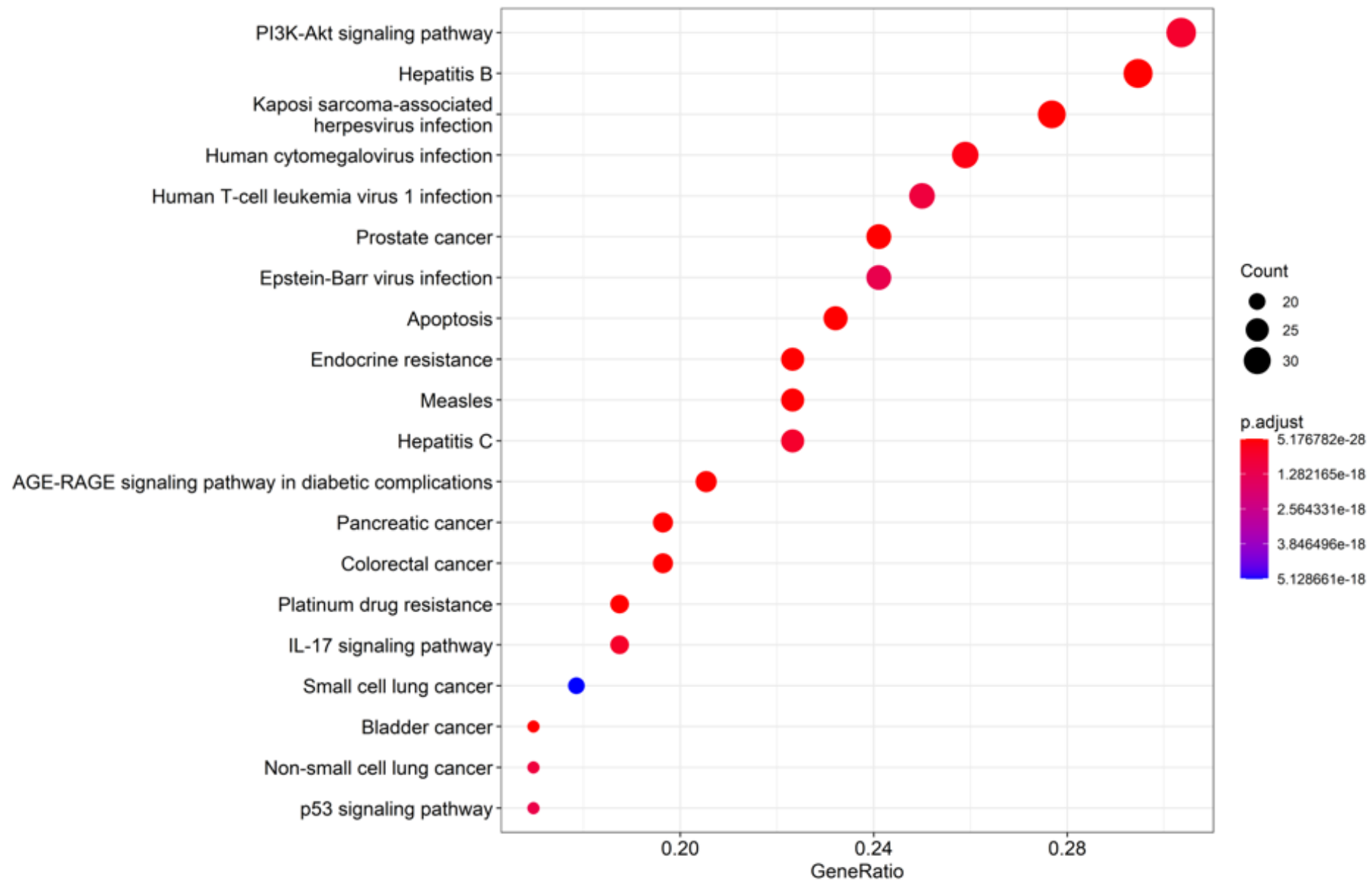

\section{Figure 10}

KEGG pathway enrichment bubble chart of the key targets of Huaijiao Diyu decoction in treatment of CRC 


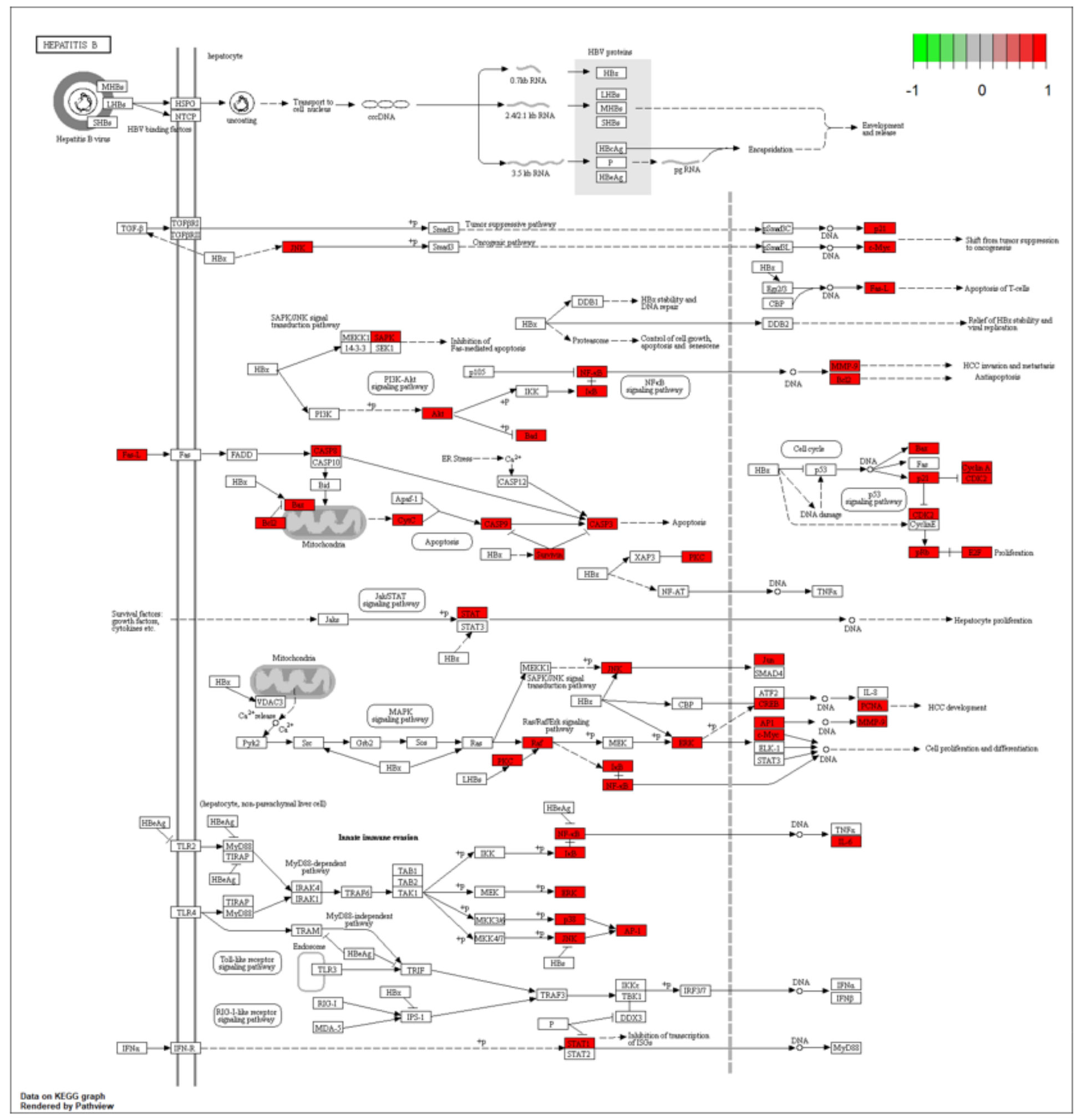

\section{Figure 11}

Distribution of target protein in the prediction pathway of Sophora japonica 


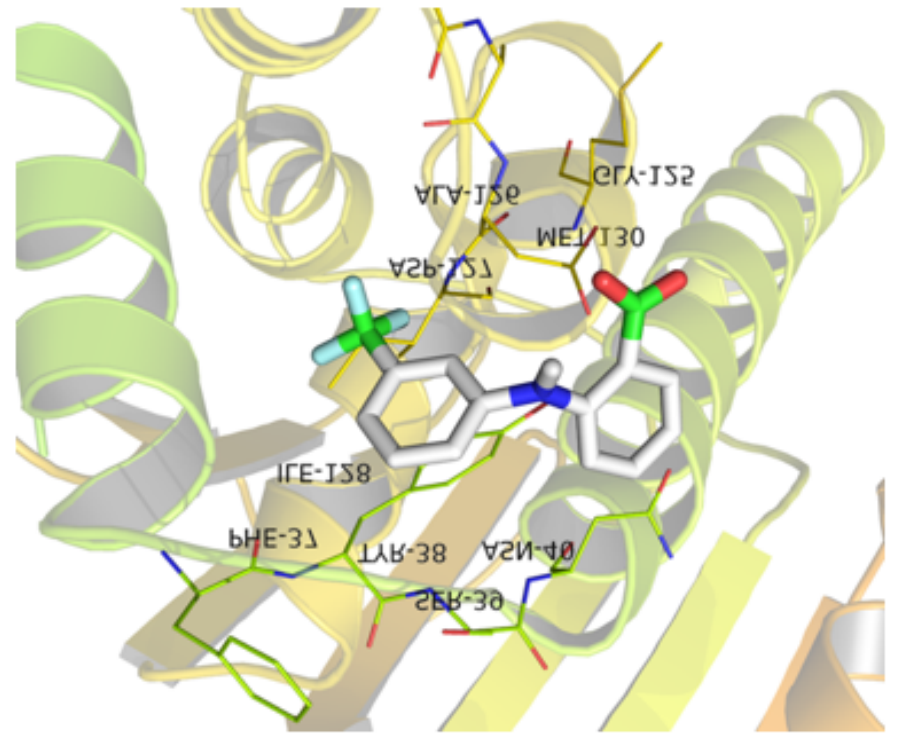

PTGS1

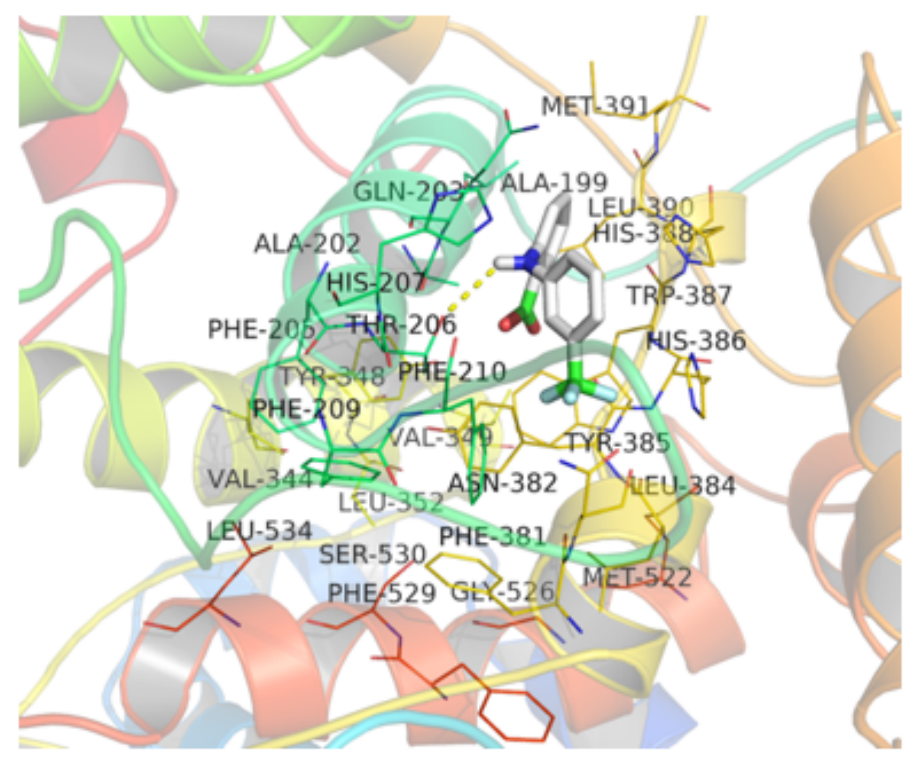

HSP90AA1

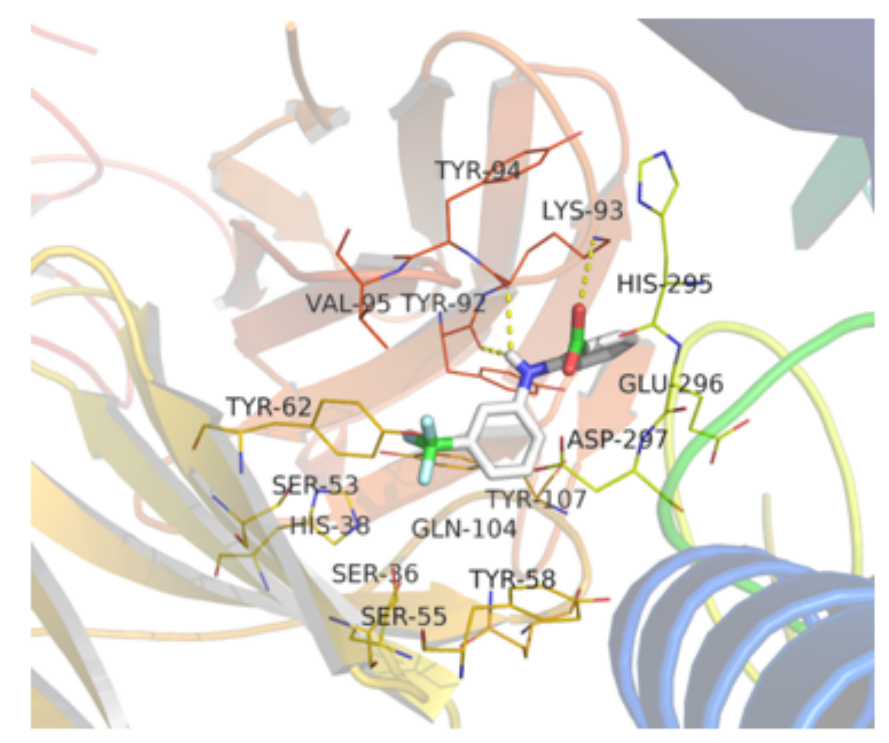

PTGS2

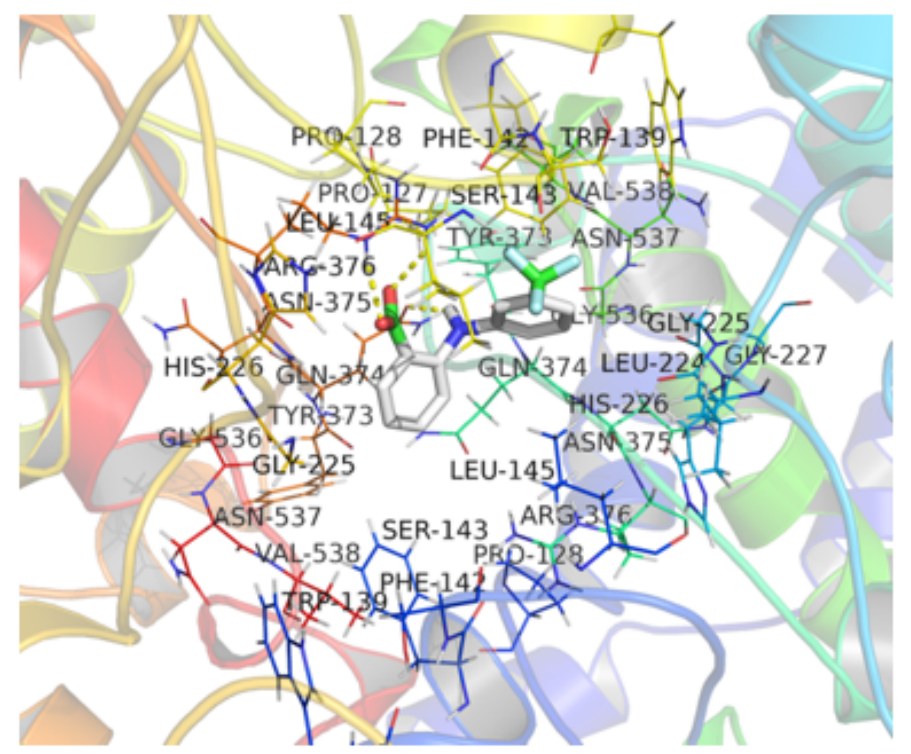

AR

Figure 12

The molecular docking diagram of quercetin and PTGS2 „Luteolin and PTGS1,Kaempfero and HSP90AA1, Wogonin and AR

\section{Supplementary Files}

This is a list of supplementary files associated with this preprint. Click to download. 
- Supplementaryinformation.docx 